\title{
MULTI-POINT MEASUREMENT OF STRUCTURAL VIBRATION USING PATTERN RECOGNITION FROM CAMERA IMAGE
}

\author{
HYEONG-SEOP JEON ${ }^{1, *}$, YOUNG-CHUL CHOI ${ }^{2}$, JIN-HO PARK ${ }^{2}$, and JONG WON PARK ${ }^{1}$ \\ ${ }^{1}$ Dept. of Information Communications Engineering, Chungnam National University \\ 220 Gung-dong Yusung-gu, Daejeon, Korea \\ ${ }^{2}$ Nuclear Technology Convergence Division, Korea Atomic Energy Research Institute (KAERI) \\ 150 Duckjin-dong Yusung-gu, Daejeon, Korea 305-353 \\ Corresponding author. E-mail : jhs200@kaeri.re.kr
}

Received March 11, 2010

Accepted for Publication October 10, 2010

Modal testing requires measuring the vibration of many points, for which an accelerometer, a gab sensor and laser vibrometer are generally used. Conventional modal testing requires mounting of these sensors to all measurement points in order to acquire the signals. However, this can be disadvantageous because it requires considerable measurement time and effort when there are many measurement points. In this paper, we propose a method for modal testing using a camera image. A camera can measure the vibration of many points at the same time. However, this task requires that the measurement points be classified frame by frame. While it is possible to classify the measurement points one by one, this also requires much time. Therefore, we try to classify multiple points using pattern recognition. The feasibility of the proposed method is verified by a beam experiment. The experimental results demonstrate that we can obtain good results.

KEYWORDS : Displacement Measurement, Pattern Recognition, Mode Analysis, Mode Shapes

\section{INTRODUCTION}

The accelerometer, laser vibrometer and displacement sensor are used to detect faults and estimate the vibration of large structures such as buildings, bridges and pipes. However, it is difficult to estimate vibration using the existing method of attaching a sensor and measuring radiation exposure at a high temperature and radioactivity where the sensor is applied. Therefore, we need a method to estimate vibration from a very great distance without attaching a sensor. A study that uses image signals generated by a camera instead of existing sensors was suggested several times $[1,2,3]$.

When using a camera at a piping structure as shown in Fig. 1, we can estimate the vibration of multiple points in one shoot. However, the point coordinates estimated from each frame should be classified to estimate the displacement of vibration at multiple points. The frame number of an image indicates a certain time, but it is necessary to know which coordinates of the multiple points detected in each frame are oriented from which point.
In this study, we suggest how to classify point coordinates and estimate the vibration occurring at the displacement of each point by applying a pattern recognizing $\mathrm{K}$-means algorithm, shooting multiple points on the

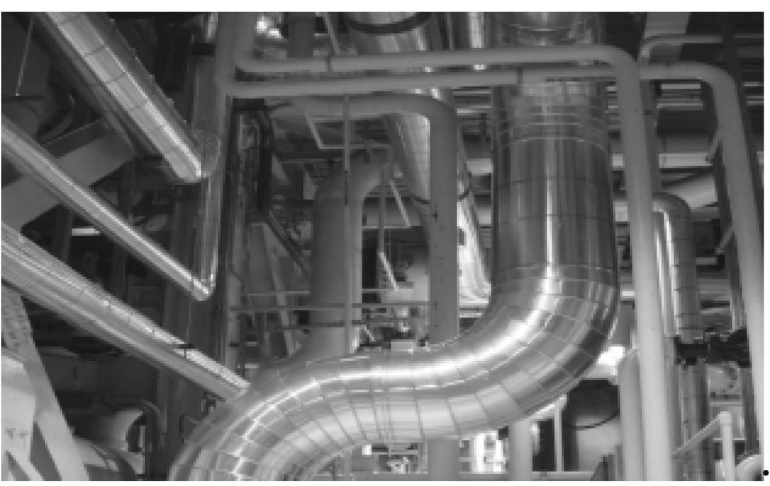

Fig. 1. Piping Structure 
structure with a camera from a great distance. The number of frames in the camera depends on the sampling frequency and the extent to which the resolution is directly related to dynamic range. It is difficult to measure highfrequency vibrations such as ultrasonic waves. However, in the case of large structures such as a bridge or a large pipe that vibrate at a low frequency, it is possible to estimate the vibration with a camera. Also, because the camera uses images, it can shoot several points on a screen at the same time. We can obtain the mode shape, as well as the frequency of vibration in this way.

After examining how to classify the coordinates measured with a camera using pattern recognition, we explain how to measure the displacement of vibration, frequency and the mode shape through tests with beams.

\section{HOW TO ESTIMATE VIBRATION WITH A CAMERA}

Images obtained from a camera were processed to obtain the conversion coordinates of selected areas, which could allow measuring of vibration displacement. This section discusses a way to measure vibration displacement through image processing $[4,5]$.

\subsection{Obtaining Images of Piping Structure Using a Camera}

To shoot the vibration of images of a pipe with a camera, mark the area to estimate a certain vibration displacement as shown in Fig. 2. Mark several points on the estimation expected positions.

\subsection{Transforming Coordinates of the Selected Point Using Image Processing}

Estimate the displacement of the selected area using the pipe image shoot image shown in Fig. 2. Figure 2(a) presents the grey image of the 1st frame. Figure 2(b) shows the images separating from the marked area from the image shown in Fig. 2(a).

$P_{1}, P_{2}$ can be separated from Fig. 2(a) and Fig. 2(b), thus making it possible to transform the coordinates of the piping structure; the related process is presented next.

Make the grey image shown in Fig. 2(a) binary coded through histogram analysis [6,7]. Acquire $P_{1}, P_{2}$ separated image in Fig. 2(b), making use of (1) Dilation and expression (2)Erosion.

$$
\mathrm{A} \oplus \mathrm{B}=\{c \mid c=a+b, a \in A, b \in B\}
$$

$$
\mathrm{A} \odot \mathrm{B}=\left\{c \mid(B)_{c} \subseteq A\right\}
$$

Find the location of $P_{1}, P_{2}$, making use of the Grassfire Algorithm labeling method [8] at Fig. 2(b) Image.

$P_{1} M(x, y), P_{2} M(x, y)$, a central coordinate of $P_{1}, P_{2}$ Area can be deducted from expression (3).

$$
x=\frac{1}{n} \sum_{i=1}^{N-1} x_{i} \quad, \quad y=\frac{1}{n} \sum_{i=1}^{N-1} y_{i}
$$

Where, $x_{i}$ and $y_{i}$ are the longitudinal \& latitudinal coordinates of the image at a labeled composition pixel.

Acquire the central coordinates of the $P_{1}, P_{2}$ area from each frame image, repeating the process from 1 to 4 for subsequent frame images.

\subsection{Partition Using Pattern Recognition}

Using the transforming coordinates of the selected area of 2.2, estimate several points at the same time and acquire each transforming coordinate. As the numbers and frames of the point coordinates increase, a certain confusion results as to which central coordinate is oriented from which point coordinate, which occurs in case they

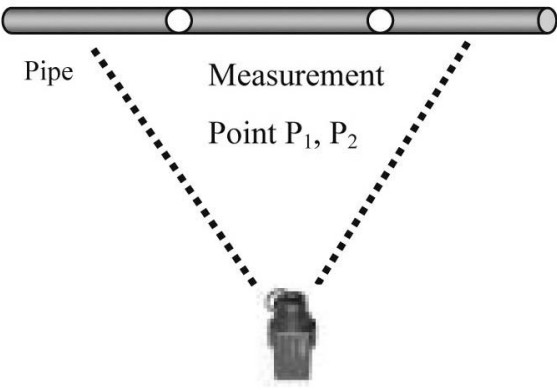

Camera

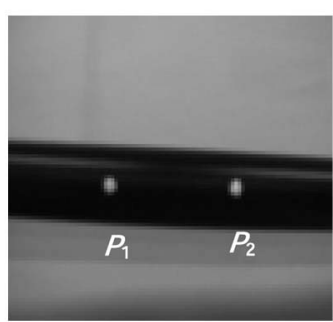

(a)

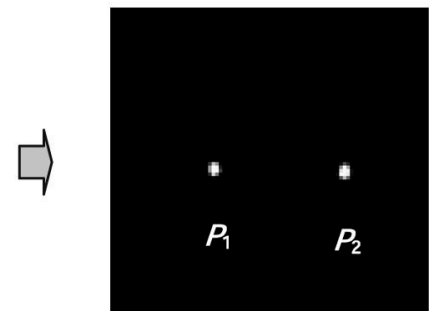

(b)
Fig. 2. Outline for Measuring Structural Vibration Using a Camera; (a) Gray Image, (b) Filtered Image 
are separated from each other. To solve this problem, separate each central coordinate automatically, making use of a pattern recognition K-means algorithm [9].

\subsubsection{K-Means Algorithm}

The K-means algorithm is widely used among nonhierarchical cluster analyses and is a simple self-study algorithm. This is a kind of analysis to find out which cluster includes each object after separating the number of clusters into M. So, it is highly suitable for the clustering of massive data.

The K-means algorithm is presented below.

1. Select M number of objects to make the property of each object the average point of each cluster.

2. Allot each object to the cluster where the averaging point approximating with each cluster locates.

3. Calculate the averaging point of each cluster.

4. If any averaging point changes, repeat the process from the 2 nd stage. If any averaging point does not change, end the clustering.

\subsubsection{Partition Making Use of K-Means Algorithm}

Acquire the central coordinates of $P 1, P 2$ and $P 3$ by each frame of the camera image. We can know it as the order in which the frames are acquired. However, any intentional decision requires knowing which coordinate is right among the $P 1, P 2$ and $P 3$ coordinates. Hence, in
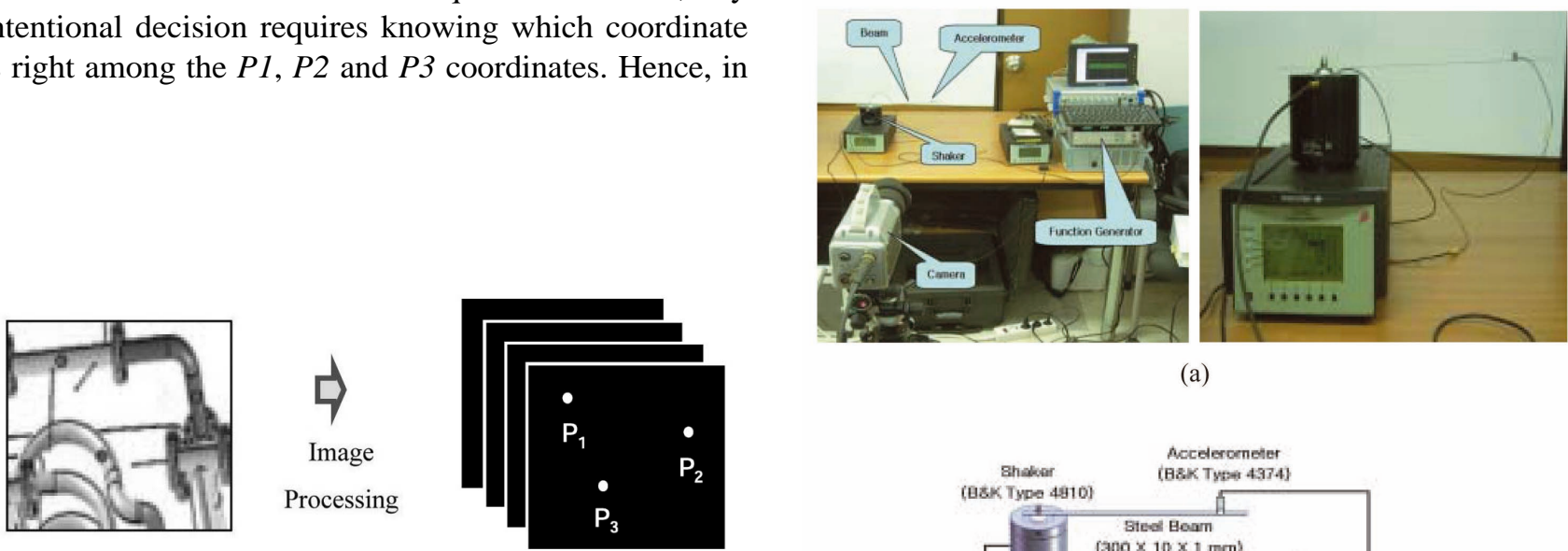

(a)

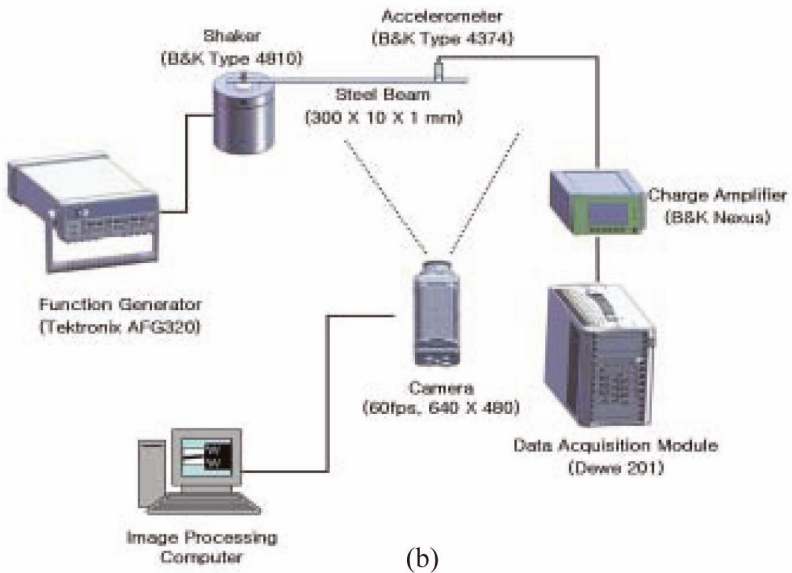

Fig. 4. Experimental Setup for Measuring Displacement Using the Camera. (a) Photo of Entire Experimental Setup. (b) Outline of Experimental Setup, where Frequency of the Shaker is $5.9 \mathrm{~Hz}$ 
generator. The camera can capture images at 60 frames per second with a resolution of $640 \times 480$. Therefore, the sampling frequency of a signal estimated from the camera is $60 \mathrm{~Hz}$.

Figure 5(a) shows a real camera image and Fig. 5(b) shows the real displacement estimated graph through this image. It can be easily seen that real displacement follows the real vibrating shape. Also, in watching the spectrum of the displacement signal at Fig. 5(c), we realized that it corresponds to $5.9 \mathrm{~Hz}$, a real frequency.

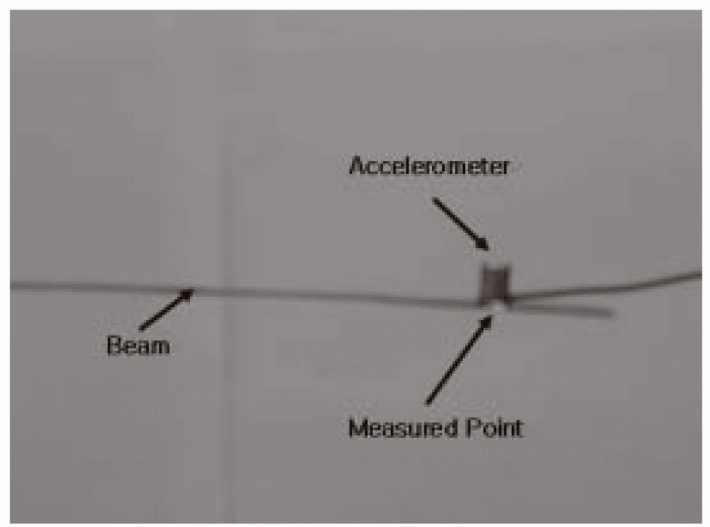

(a)

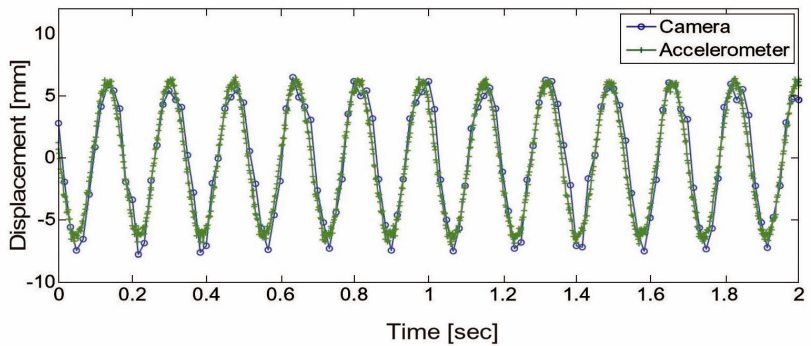

(b)

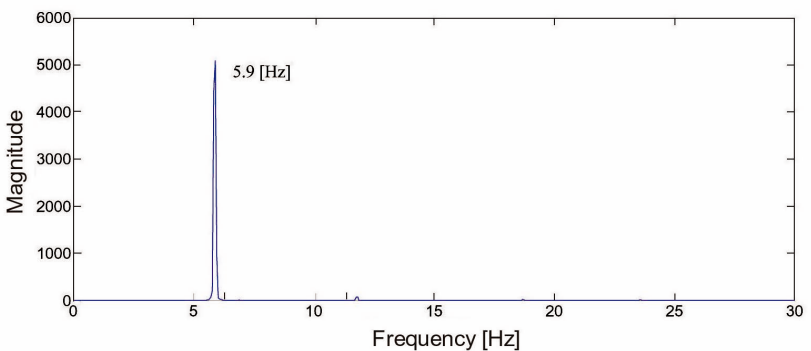

(c)

Fig. 5. Experimental Results. (a) Image of Measurement Point. (b) Displacement at the Accelerometer Location. '+'Green Line Means True Displacement from Accelerometer and 'o'Blue Line is Measured Result from Image Processing. (c) Power Spectrum from Camera Image Signal Main Frequency $5.9 \mathrm{~Hz}$ Coincides with Exciting Frequency of the Function Generator

\subsection{Modal Test Experiment}

One of the most important advantages in measuring displacement with a camera is that it can measure many points simultaneously, making it possible to measure mode shapes as well.

Figure 6 describes an experimental setup to measure the mode shapes of a beam. The material of the beam is SUS304, and its dimensions are $700 \mathrm{~mm} \times 25 \mathrm{~mm} \times 1 \mathrm{~mm}$.

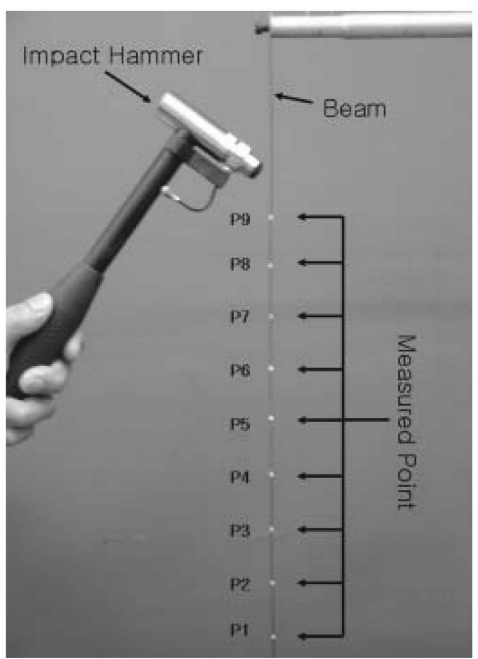

Fig. 6. Measurement Experiment on Beam Mode Shapes

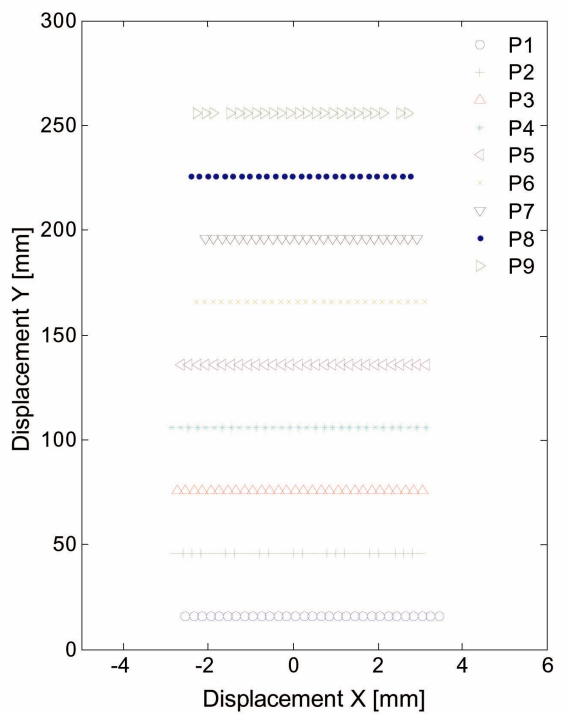

Fig. 7. Coordinates Separation Using K-Means Algorithm from Experiment, where 'o'Point 1, '+'Point 2, .., ' $\triangleright$ 'Point 9 
Nine points are selected as measurement points as shown in Fig. 6. The beam was excited using an impact hammer. At this time, a total of 7 second of time camera images are stored at 400 frames per second.

By using the $\mathrm{K}$-means algorithm, the automatic dissociated result coordinates in which it measures in 9 points is shown through 9 groups in Fig. 7. In this way, in one camera video image, many points can be measured and classified at the same time.

The displacement signal of each point in which it classifies through pattern recognition is shown in Fig. 8. To obtain natural frequency and mode shapes, we performed a Fourier transform of the displacement signals at each point. Figures 9 and 10 show the magnitude and phase of the measured signals. The natural frequencies are $1 \mathrm{~Hz}, 5.3 \mathrm{~Hz}, 14 \mathrm{~Hz}$ and $28 \mathrm{~Hz}$.

Measured displacement [mm]
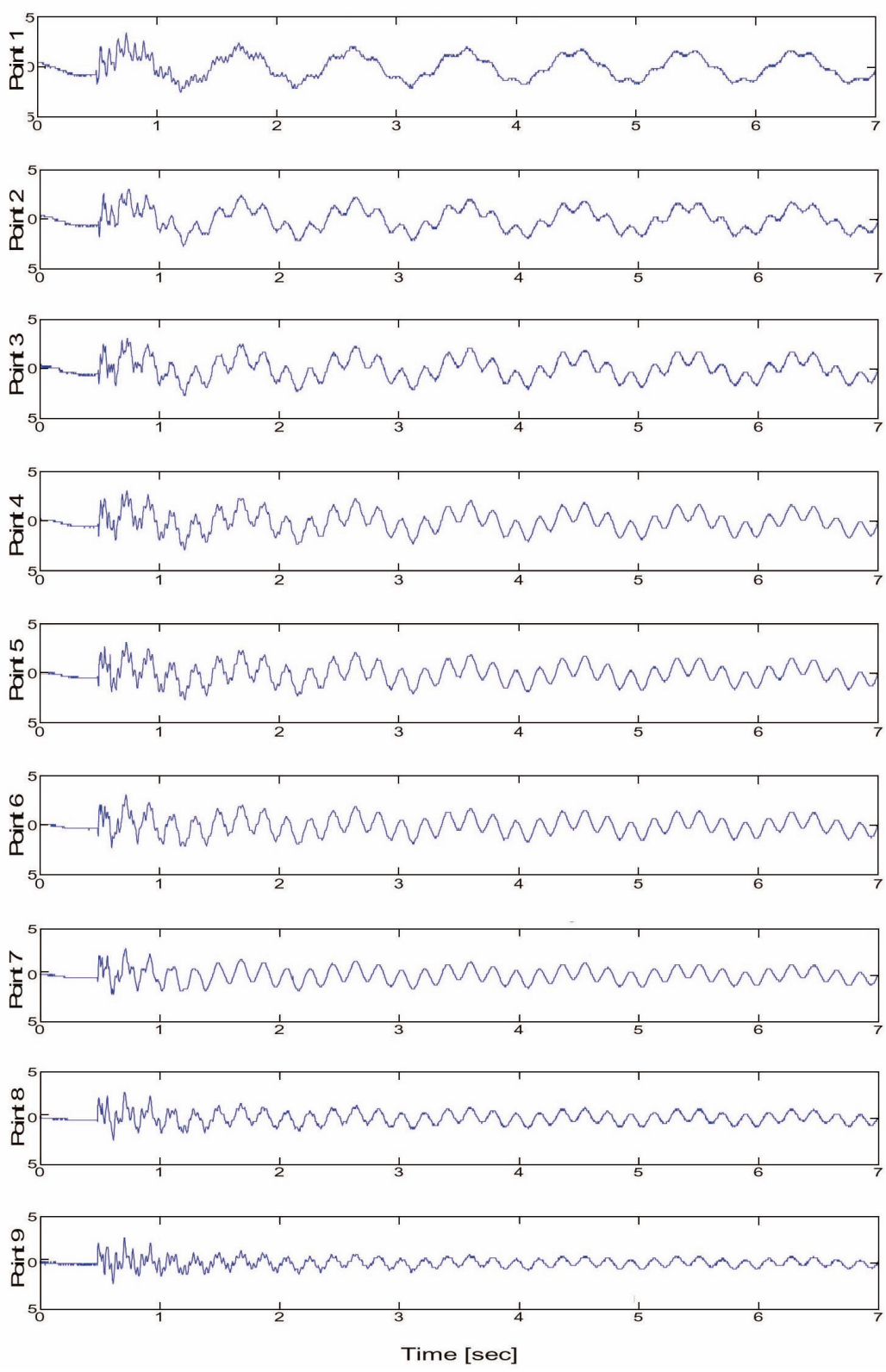

Fig. 8. Measured Displacements at Nine Measurement Points 


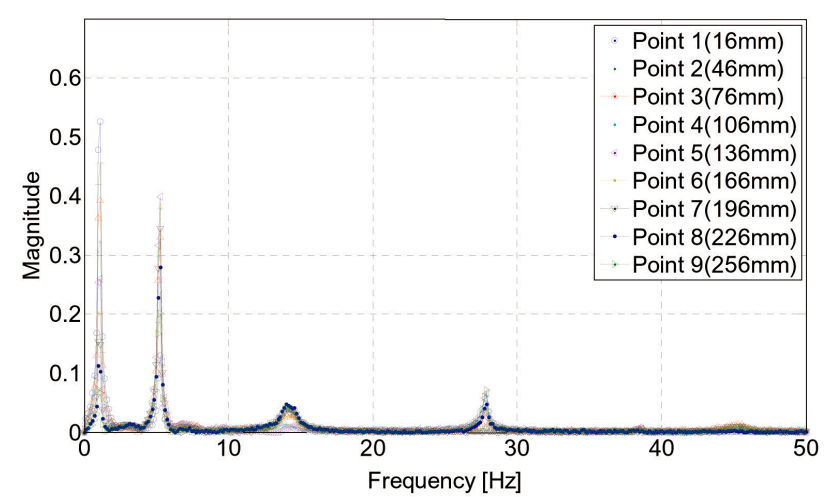

Fig. 9. Spectrum Magnitudes of Measured Signals. Natural Frequencies are $1 \mathrm{~Hz}, 5.3 \mathrm{~Hz}, 14 \mathrm{~Hz}$, and $28 \mathrm{~Hz}$

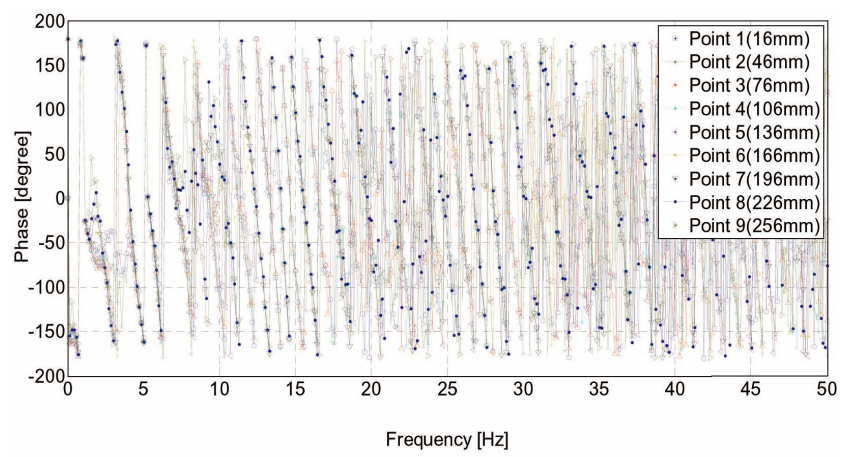

Fig. 10. Spectrum Phases of Measured Signals

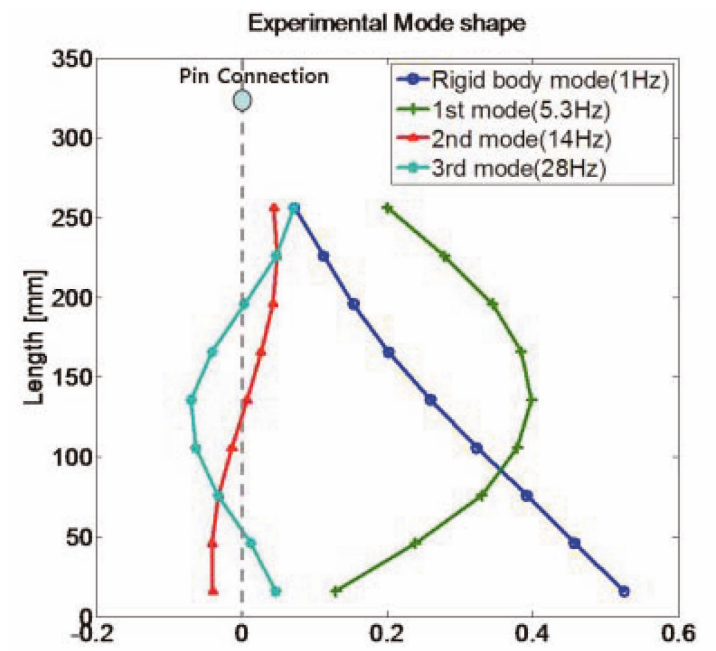

Fig. 11. Experimental Mode shapes. 'o’Blue Line, ‘+'Green Line, ' $\triangle$ 'Red Line and '*'Sky Blue Line Mean Rigid Body Mode, $1^{\text {st }}$ Mode, $2^{\text {nd }}$ Mode and $3^{\text {rd }}$ Mode Shape
Magnitudes and phases according to the natural frequency are arranged in Table 1.

Using Table 1, the natural mode shapes can be plotted as shown in Fig. 11. To compare with the exact solution, we derived mode shapes theoretically

The tested beam is a simple uniform "pinned-free" beam, so we can derive the natural frequencies and mode shapes theoretically. To determine the natural frequencies and mode shapes, we employ separation of variables. Thus we let

$$
y(x, t)=Y(x) T(t)
$$

and substituting in the Bernoulli-Euler theory of beams gives [10]

$$
\begin{aligned}
T(t)= & A \cos \omega t+B \sin \omega t \\
Y(x)= & C_{1}(\cos \beta x+\cosh \beta x)+C_{2}(\cos \beta x-\cosh \beta x) \\
& +C_{3}(\sin \beta x+\sinh \beta x)+C_{4}(\sin \beta x-\sinh \beta x)
\end{aligned}
$$

Because the boundary condition of the tested beam is pinned free we can let

$$
\begin{aligned}
& Y(0)=\frac{d^{2} Y(0)}{d x^{2}}=0 \\
& \frac{d^{2} Y(l)}{d x^{2}}=\frac{d^{3} Y(l)}{d x^{3}}=0
\end{aligned}
$$

Using the solution form eq. (5) in these boundary conditions gives

$$
\begin{aligned}
& C_{1}=C_{2}=0 \\
& C_{3}=\frac{\sinh \beta l+\sin \beta l}{\sinh \beta l-\sin \beta l} C_{4}
\end{aligned}
$$

The mode shapes are then

$$
\begin{aligned}
Y(x)= & \frac{2 C_{4}}{\sinh \beta l-\sin \beta l} \\
& \{\sinh l \cdot \sin \beta x+\sin \beta l \cdot \sinh \beta x\}
\end{aligned}
$$


Table 1. Magnitude and Phase at Each Measured Point

\begin{tabular}{|c|c|c|c|c|c|c|c|c|}
\hline & \multicolumn{2}{|c|}{ Rigid body mode $(1 \mathrm{~Hz})$} & \multicolumn{2}{|c|}{$1^{\text {st }}$ mode $(5.3 \mathrm{~Hz})$} & \multicolumn{2}{|c|}{$2^{\text {nd }}$ mode $(14 \mathrm{~Hz})$} & \multicolumn{2}{|c|}{$3^{\text {rd }}$ mode $(28 \mathrm{~Hz})$} \\
\hline & Mag. & Phase & Mag. & Phase & Mag. & Phase & Mag. & Phase \\
\hline $\mathrm{P} 1$ & 0.5261 & $0^{\circ}$ & 0.1300 & $0^{\circ}$ & 0.0393 & $180^{\circ}$ & 0.0464 & $0^{\circ}$ \\
\hline $\mathrm{P} 2$ & 0.4571 & $0^{\circ}$ & 0.2393 & $0^{\circ}$ & 0.0411 & $180^{\circ}$ & 0.0125 & $0^{\circ}$ \\
\hline P3 & 0.3929 & $0^{\circ}$ & 0.3307 & $0^{\circ}$ & 0.0311 & $180^{\circ}$ & 0.0321 & $180^{\circ}$ \\
\hline P4 & 0.3229 & $0^{\circ}$ & 0.3786 & $0^{\circ}$ & 0.0136 & $180^{\circ}$ & 0.0625 & $180^{\circ}$ \\
\hline P5 & 0.2600 & $0^{\circ}$ & 0.3989 & $0^{\mathbf{o}}$ & 0.0079 & $0^{\circ}$ & 0.0696 & $180^{\circ}$ \\
\hline P6 & 0.2025 & $0^{\circ}$ & 0.3839 & $0^{\circ}$ & 0.0268 & $0^{\circ}$ & 0.0404 & $180^{\circ}$ \\
\hline P7 & 0.1536 & $0^{\circ}$ & 0.3446 & $0^{\circ}$ & 0.0429 & $0^{\circ}$ & 0.0036 & $0^{\mathbf{o}}$ \\
\hline P8 & 0.1129 & $0^{\circ}$ & 0.2786 & $0^{\circ}$ & 0.0482 & $0^{\circ}$ & 0.0471 & $0^{\circ}$ \\
\hline P9 & 0.0732 & $0^{\circ}$ & 0.2000 & $0^{\circ}$ & 0.0446 & $0^{\circ}$ & 0.0714 & $0^{\circ}$ \\
\hline
\end{tabular}

And the resulting frequency equation is

$$
\tan \beta l=\tanh \beta l
$$

The first few roots are given by

$$
\beta_{1} l=3.927, \beta_{2} l=7.069, \beta_{3} l=10.210
$$

Figure 12 shows the theoretical mode shapes plotted by using eq. (8) (10). Theoretical mode shapes coincide with the experimental result of Fig. 11 in the yellow box. Therefore, natural frequency $1 \mathrm{~Hz}$ is a rigid body mode, $5.3 \mathrm{~Hz}$ is $1^{\text {st }}$ mode, $14 \mathrm{~Hz}$ is $2^{\text {nd }}$ mode, and $28 \mathrm{~Hz}$ is $3^{\text {rd }}$ mode.
Theoretical mode shape

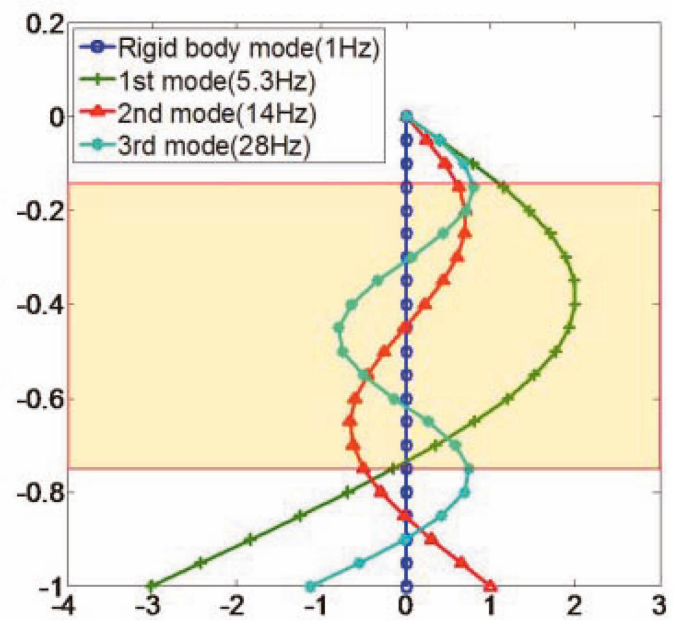

Fig. 12. Theoretical Mode shapes. 'o'Blue Line, '+'Green Line, ' $\triangle$ 'Red Line and '*'Sky Blue Line Mean Rigid Body Mode, $1^{\text {st }}$ Mode, $2^{\text {nd }}$ Mode and $3^{\text {rd }}$ Mode Shape. The Mode Shape Coincides with Experimental Results in the Yellow Box 
The conventional methods of mode analysis must adhere to the accelerometer at all measurement points and acquire the signals. Therefore, there exists a considerable disadvantage in terms of measurement time and effort when there are many measurement points. However, the proposed method can measure the vibration of all points in one measurement because it uses camera imagery. Moreover, it can be increased as much as the desired number of measurements.

\section{CONCLUSION}

This paper proposes a technique for measuring vibration at multiple points using a camera image. To measure multiple points under current methods, the measurement points must be classified one by one for each frame, but this requires much time. However, the proposed method can classify the measurements automatically. This is because it uses the K-means algorithm. Also, because it is possible to estimate the displacement of several points in one shooting of the image for certain complicated piping or structures, we can easily find the pattern of vibration mode of a structure through a test. To verify the proposed method, we have performed beam experiments to obtain the natural mode shapes. Experiments show that measured mode shapes coincide with theoretical mode shapes obtained from an exact solution.

As the number of camera frames responds to the frequency and the resolution influences on the error of the displacement, we can estimate high frequency without much error when using a camera equipped with high resolution and the ability to capture many frames, e.g., a camera that can shoot 60 and 400 frames per second with a resolution of $640 \times 480$. As the sampling frequency of the signal estimated from cameras is $60 \mathrm{~Hz}$ and $400 \mathrm{~Hz}$, it is possible to estimate vibration without aliasing if the vibration is below $40 \mathrm{~Hz}$

\section{REFERENCES}

[1] Lee, S. B. and Kwak, M. K., 2000, "Development of Vibration Measurement Technique Using the Image Processing" Proceedings of the KSNVE Annual Autumn Conference, pp. 327 329.

[2] Kim, K. Y. and Kwak, M. K., 2005, "Measurement of Large-amplitude and Low-frequency Vibrations of Structures Using the Image Processing Method", Transactions of the Korean Society for Noise and Vibration Engineering, Vol. 15, No. 3, pp. 329 333. 2005.

[3] Wahbeh, A. M., Caffrey, J. P., and Masri, S. F., “A Vision-based Approach for the Direct Measurement of Displacements in Vibrating Systems." Smart Structures and Materials, Vol. 12, 2003, pp. 785 794.

[4] James R. Parker, Algorithms for Image Processing and Computer Vision, John Wiley \&Sons, New York, 1997

[ 5 ] R. C. Gonzalez and R. E. Woods, Digital Image Processing, Addison Wesley, 1992.

[6] Chowdhury, M. H. and W. D. Little, "Image Thresholding Techniques", 1995, IEEE Pacific Rim Conference on Communications, Computers, and Signal Processing, pp. 585-589.

[7] Otsu, N., 1979, "A Threshold Selection Method from Gray-level Histograms", IEEE Trans. Syst. Man Cybern. 9, pp. 62-66.

[8] I.Pitas "Digital Image Processing Algorithms and Applications ", 2000, John Wiley \& Sons, Inc, pp.300 303.

[9] J. B. MacQueen (1967): "Some Methods for classification and Analysis of Multivariate Observations, Proceedings of 5-th Berkeley Symposium on Mathematical Statistics and Probability", Berkeley, University of California Press, 1:281-297

[10] Karl F. Groff, Wave motion in elastic solids, 1991, dover publications, INC., pp.140-158 